\title{
Antagonistic Effects of Doublecortin and MARK2/Par-1 in the Developing Cerebral Cortex
}

\author{
Tamar Sapir, ${ }^{1}$ Anat Shmueli, ${ }^{1}$ Talia Levy, ${ }^{1}$ Thomas Timm, ${ }^{3}$ Michael Elbaum, ${ }^{2}$ Eva-Maria Mandelkow, ${ }^{3}$ and Orly Reiner ${ }^{1}$ \\ Departments of ${ }^{1}$ Molecular Genetics and ${ }^{2}$ Materials and Interfaces, The Weizmann Institute of Science, 76100 Rehovot, Israel, and ${ }^{3}$ Max Planck Unit for \\ Structural Molecular Biology, 22607 Hamburg, Germany
}

\begin{abstract}
Abnormal neuronal migration is manifested in brain malformations such as lissencephaly. The impairment in coordinated cell motility likely reflects a faulty mechanism of cell polarization or coupling between polarization and movement. Here we report on the relationship between the polarity kinase MARK2/Par-1 and its substrate, the well-known lissencephaly-associated gene doublecortin $(D C X)$, during cortical radial migration. We have previously shown using in utero electroporation that reduced MARK2 levels resulted in multipolar neurons stalled at the intermediate zone border, similar to the phenotype observed in the case of DCX silencing. However, whereas reduced MARK2 stabilized microtubules, we show here that knock-down of DCX increased microtubule dynamics. This led to the hypothesis that simultaneous reduction may alleviate the phenotype. Coreduction of MARK2 and DCX resulted in a partial restoration of the normal neuronal migration phenotype in vivo. The kinetic behavior of the centrosomes reflected the different molecular mechanisms activated when either protein was reduced. In the case of reducing MARK2 processive motility of the centrosome was hindered, whereas when DCX was reduced, centrosomes moved quickly but bidirectionally. Our results stress the necessity for successful coupling between the polarity pathway and cytoplasmic dynein-dependent activities for proper neuronal migration.
\end{abstract}

Key words: neuronal migration; DCX; MARK2/Par-1; lissencephaly; microtubules; in utero electroporation

\section{Introduction}

Coordinated neuronal migration depends on development and maintenance of cell polarization. We have recently demonstrated a role for the polarity kinase MARK2/Par-1 in neuronal migration (Sapir et al., 2008). MARK2/Par-1 phosphorylates doublecortin (DCX) (Schaar et al., 2004), another microtubuleassociated protein (MAP), mutations in which cause X-linked lissencephaly (Francis et al., 1999; Gleeson et al., 1999; Horesh et al., 1999). We hypothesize that interaction between MARK2/ Par-1 and DCX may regulate cortical radial migration. These proteins are likely to affect microtubule dynamics, which are tightly balanced during neuronal migration.

MARK2/Par-1 plays an important role in regulation of cy-

Received May 26, 2008; revised Sept. 27, 2008; accepted 0ct. 22, 2008

This work was supported in part by the Israeli Science Foundation (Grant no. 270/04 and equipment grant to 0.R.), Foundation Jérôme Lejeune, Minerva Foundation with funding from the Federal German Ministry for Education and Research, the German-Israeli collaboration Grant Gr-1905, a grant from the March of Dimes (\#6-FY07-388), a grant from the Paul Godfrey Research Foundation in Childrens' Diseases, the Benoziyo Center for Neurological Diseases, the Kekst Center, the Forcheimer Center, a Weizmann-Pasteur collaborative grant, a research grant from the Michigan Women of Wisdom Fund to support Weizmann Women Scientists, support from Mr. Maurice Janin, the Jewish communal fund Albert Einstein College of Medicine of Yeshiva University, and the David and Fela Shapell Family Center for Genetic Disorders Research. 0.R. is an incumbent of the Berstein-Mason Professorial Chair of Neurochemistry. Work of M.E. is made possible in part by the historic generosity of the Harold Perlman family. This work has also been supported in part by the Deutsche Forschungsgemeinschaft (to E.M.), and the Max-PlanckGesellschaft. We thank Drs. Michel Bornens, Trina Schroer, Hillary Voet, Salvador Martinez, Ari Elson, Eli Arama, Juergen Wehland, Gregg Gundersen, Franck Polleux, Tony Barnes, Joe LoTurco, Niels Galjard, and Eran Hornstein for useful comments and/or reagents.

Correspondence should be addressed to Orly Reiner, Department of Molecular Genetics, The Weizmann Institute of Science, 76100 Rehovot, Israel. E-mail: orly.reiner@weizmann.ac.il.

DOI:10.1523/JNEUROSCI.2363-08.2008

Copyright $\odot 2008$ Society for Neuroscience $\quad 0270-6474 / 08 / 2813008-06 \$ 15.00 / 0$ toskeletal dynamics (Timm et al., 2006), in particular microtubule dynamics (Drewes et al., 1997; Drewes et al., 1998; Sapir et al., 2008). Following MARK2 phosphorylation its MAP substrates detach from tubulin polymers. Known substrates include tau, MAP-2/MAP-4, and DCX (Drewes et al., 1997; Schaar et al., 2004). DCX binds to microtubules in a unique position in between the protofilaments, and stabilizes them (Moores et al., 2004). Therefore, we raised the hypothesis that reduction in the cellular expression of DCX may affect microtubule dynamics. In addition, MARK2/Par-1 and DCX are suggested to play an important role in regulation of cell polarity. A genetic interaction between the dynein pathway and the polarity pathway is suggested by the fact that orthologs of LIS1, cytoplasmic dynein and DCX affect the first asymmetric cell division in Caenorhabditis elegans (Gönczy et al., 2000). LIS1 (Reiner et al., 1993) and DCX (des Portes et al., 1998; Gleeson et al., 1998), both of which are implicated in neuronal migration disorders, regulate centrosomal-nuclear coupling in a dynein-dependent pathway (Shu et al., 2004; Tanaka et al., 2004a; Tsai et al., 2005, 2007). Furthermore, reduction in MARK2 affects centrosomal movement in radially migrating neurons (Sapir et al., 2008). LIS1 and its interacting protein Ndel1 regulate Cdc42 (Kholmanskikh et al., 2003, 2006; Shen et al., 2008), which impacts a repertoire of targets including PAR-6, aPKC, PAR-3 and PAK5 (Govek et al., 2005). Based on the above observations, we hypothesized that MARK2/Par-1 and its substrate DCX may cooperate during neuronal migration as key node proteins joining the polarity and the dynein pathways.

Reduction in cellular DCX is expected to destabilize microtubules, while reduction in the cellular level of MARK2 should 

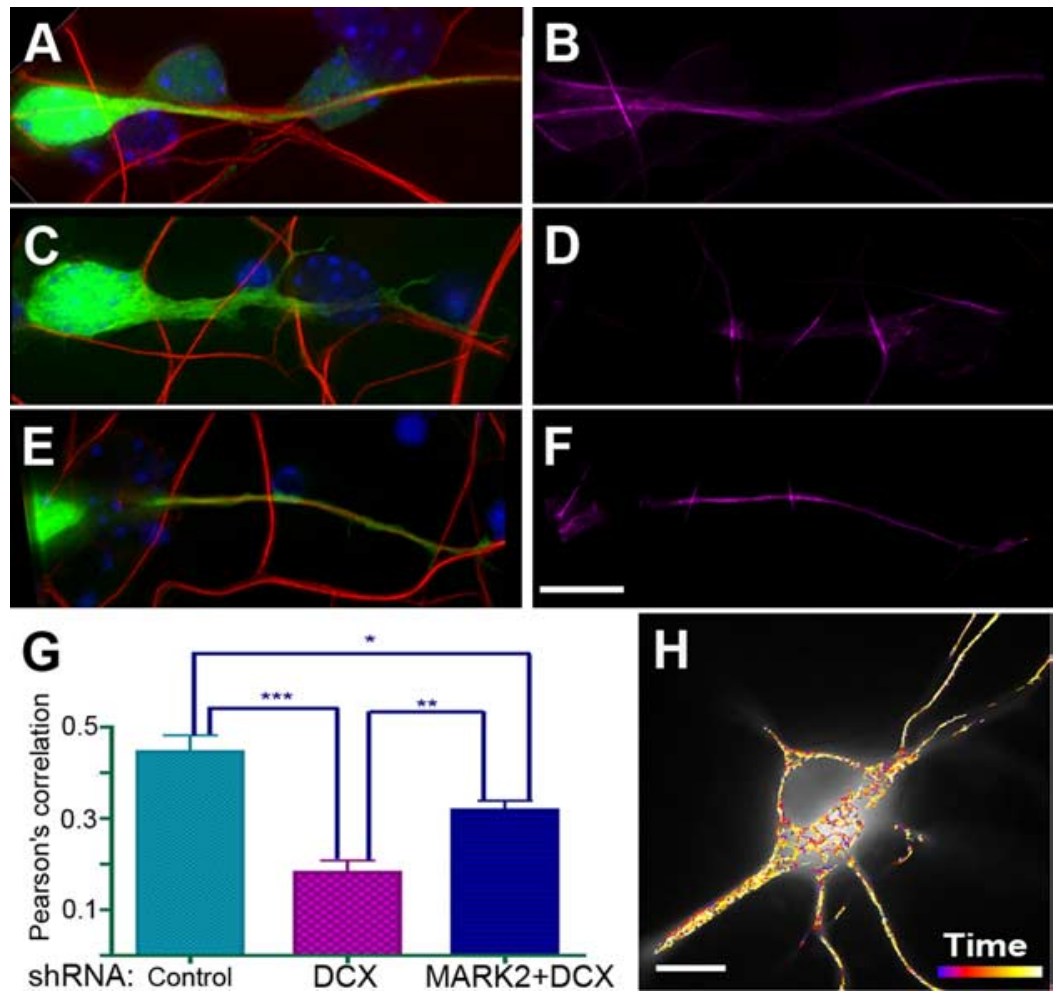

Figure 1. Reduction in DCX destabilizes microtubules in vitro. $\boldsymbol{A}-\boldsymbol{F}$, Microtubule stability in cerebellar neurons. $\boldsymbol{A}, \boldsymbol{C}, \boldsymbol{E}$, Transfected neurons are labeled with GFP (green), immunostained with antidetyrosinated tubulin antibodies (red), and the nuclei labeled with DAPI (blue). $\boldsymbol{B}, \boldsymbol{D}, \boldsymbol{F}$, Colocalization between GFP and detyrosinated tubulin is shown in a false purple color. The treatments include control shRNA $(\boldsymbol{A}, \boldsymbol{B}), \mathrm{DCX}$ shRNA $(\boldsymbol{C}, \boldsymbol{D})$, and a combination of DCX and MARK2 shRNA $(\boldsymbol{E}, \boldsymbol{F})$. Scale bar, $\boldsymbol{F}, 10$ $\mu \mathrm{m}$. G, Quantification of Pearson's correlation of the colocalization between GFP and detyrosinated tubulin was calculated using Imaris colocalization measurement tool and subjected to ANOVA statistical analysis. ${ }^{*} p<0.05,{ }^{* *} p<0.01,{ }^{* * *} p<0.001$; error bars indicate \pm SEM. $\boldsymbol{H}$, Hippocampal neurons transfected with indicated shRNA and EB3-GFP tracked using Imaris cell tracking system. A representative neuron with lines shown in different colors marking the advance of individual tracks over time. Scale bar, H, $5 \mu \mathrm{m}$.

stabilize microtubules by alleviating its effect on DCX. Subsequently, coreduction of both proteins could potentially result in partial restoration of a normal migration pattern in vivo, as shown here. Reduction in MARK2 or DCX also affected processive centrosomal movement, a process subject to dynein regulation. The molecular mechanisms underlying nonprocessive motility differed in an essential manner. Whereas reduction in MARK2 resulted in significantly less motile centrosomes, reduction in DCX enhanced centrosomal movement in both forward and backward orientations, resulting however in reduced net processivity. These results demonstrate intercalation between the polarity pathway with the cytoplasmic dynein-mediated neuronal migration pathway and highlight the accurate coupling required for radial migration in the cerebral cortex.

\section{Materials and Methods}

Neuronal cultures and in utero electroporation. Hippocampal and cerebellar neurons were prepared as described previously (Brann et al., 2002; Gdalyahu et al., 2004) and transfected by the Amaxa protocol. Following electroporation, neurons were stained or imaged live as described previously (Sapir et al., 2008) and analyzed using the Delta-Vision system package, or Imaris software. In case of EB3-GFP transfections, eight cells with a total of 11,375 tracks were analyzed from control cells, and six cells with a total of 9145 tracks from DCX shRNA-treated cells. In utero electroporations were conducted as described previously (Sapir et al., 2008). Additional information is provided in supplemental material, available at www.jneurosci.org.

Quantitative measurements. The colocalization program in the Soft-
Worx suite was used to determine the degree of colocalization and the Pearson coefficient of correlation was calculated. The program generates a new product image of two channels after subtracting a threshold value for each.

Neuronal migration following in utero electroporation was analyzed from at least three different embryos and at least eight different sections. Sections were stained with DAPI, and the different brain areas were identified. Comparison between different treatments of the ratio of GFP-positive cells reaching the superficial area of the cortical plate $(\mathrm{CP})$ was analyzed using one-way ANOVA, and Tukey's post test was performed using GraphPad Prism version 4.00 for Macintosh (GraphPad Software).

To evaluate centrosomal displacement, centrosomal locations were extracted by the transfected centrin fluorescence. In cases where the centrioles split, each individual centriole was analyzed. The route taken by each centrosome was computationally rotated to a horizontal orientation, and the displacement along the axis was calculated for successive time points. Frames were recorded at 2 min intervals.

\section{Results}

The hypothesis that reduction of DCX may result in impaired microtubule stability was tested first by immunostaining of transfected primary cerebellar and hippocampal neurons (Fig. 1). Cotransfection was performed with small hairpin RNA directed against DCX or a control shRNA or a combination of DCX and MARK2 shRNA and an expression plasmid for GFP as a marker. Cells were stained using antidetyrosinated $\alpha$-tubulin antibodies, a marker of stabilized microtubules (Fig. $1 A-F)$. Microtubule stabilization was reduced in DCX shRNAtreated neurons (Fig. 1, compare $C, D, A, B$ ). This was evaluated by comparing the degree of colocalization of GFP with the immunolabeled detyrosinylated tubulin between DCX shRNA- and control shRNA-transfected cells. The degree of colocalization was quantified by the Pearson coefficient of correlation (Fig. 1G) (in primary cerebellar neurons Student's $t$ test $p<0.0001$, mean \pm SEM of control $0.44 \pm 0.035, n=14$, mean \pm SEM of DCX shRNA $0.18 \pm 0.02, n=23$ ) and was found to be statistically significant. Similar results were obtained in cultured hippocampal neurons (data not shown). We have previously demonstrated that MARK2 reduction increased microtubule stability (Sapir et al., 2008). Therefore, we postulated that coreduction of DCX and MARK2 may result in increased stability of microtubules in comparison with the single reduction of DCX. Coreduction of DCX and MARK2 resulted in an observed increase in microtubule stability (Fig. 1, compare E, F, C,D). The degree of colocalization was quantified as described above, and the increase in microtubule stability in comparison with DCX shRNA treatment was found to be statistically significant, although it was still lower than control levels (Fig. 1G) (ANOVA analysis, DCX shRNA vs the double treatment $p<0.01$, control vs the double treatment $p<0.05$, DCX shRNA vs control $p<0.001)$. These results suggest that coreduction of DCX and MARK2 converges at the level of microtubule stability.

We next examined microtubule dynamics in live cells by mea- 
suring the duration of EB3-GFP residence on microtubule tips (Fig. $1 H$ ) in cultured hippocampal neurons. Each track in the EB3 movie is encoded by a rainbow color representing time. Neurons transfected with DCX shRNA exhibited less stable microtubules implied by the shorter duration of EB3 on the tips of microtubules (Student's $t$ test $p<0.0001$, mean \pm SEM of control $7.364 \pm 0.11, n=11,373$, mean \pm SEM of DCX shRNA $6.398 \pm 0.11, n=$ 9143). An inverse correlation was noted for the rate of microtubule polymerization; microtubules polymerized faster in the absence of DCX than in control cells (Student's $t$ test $p<0.0001$, mean \pm SEM of control $0.3406 \pm 0.002, n=11,373$, mean \pm SEM of DCX shRNA $0.3974 \pm$ $0.002, n=9143)$. Collectively, the data are consistent with altered microtubule growth and stability, indicating that DCX may be an important regulator of microtubule dynamics.

In vivo, reducing DCX by in utero electroporation resulted in a pronounced inhibition of neuronal migration in comparison with control $(p<0.0001)$ (Fig. 2, compare $B, A$ ). The phenotype was similar to that of a previous report (Bai et al., 2003). Notably, shRNA reduction of MARK2 had a similar effect (Fig. 2C). We noted the presence of a typical band of neurons stalled at the intermediate zone (IZ) border in the cortical plate, where it has been demonstrated that multipolar neurons accumulate (Bai et al., 2003; Sapir et al., 2008). Coreduction of MARK2 and DCX resulted in a partial but significant improvement in neuronal migration in comparison with the individual reduction in either DCX or MARK2 (Fig. 2D,E) (statistical analysis by ANOVA, coreduction in comparison with DCX shRNA $p<0.001$, coreduction in comparison with MARK2 shRNA $p<0.05)$. Most strikingly, there was a notable decrease in the number of neurons stalled at the IZ boundary. These findings suggest that DCX may be an important MARK2 substrate in the developing cortex.

DCX has been shown to complex with dynein. The lack of Lis 1 resulted in an increase in the distance between the nucleus and the centrosome; expression of DCX in neurons lacking one Lis1 allele compensated for the observed phenotype (Tanaka et al., 2004a). We have previously shown that reduction in MARK2 levels suppressed movement of centrosomes in migrating cortical neurons, resulting in heterotropic multipolar cells at the IZ boundary. Therefore we investigated the dynamic behavior of the centrosome in migrating neurons with reduced DCX. Distinct modes of centrosomal motility during radial neuronal migration can be identified in time-lapse movies of the brain slices (Fig. $3 A-G$; supplemental movies 1-4, available at www.jneurosci. org as supplemental material). Control centrosomes labeled by centrin-RFP move continuously in a forward direction (Fig. $3 A, B, G$ ), as reported previously (Tsai et al., 2007; Sapir et al., 2008). In the presence of DCX shRNA, in contrast, centriole splitting and random motility of centrosomes were evident in many cells (Fig. 3C,D, G; supplemental movies 1,2, available at www. jneurosci.org as supplemental material). In several cases the centrosome traveled from the leading edge all the way to the rear of the cell. This extraordinary motility has not been previously observed in migrating cortical neurons. Interestingly, coexpression of MARK2 shRNA with DCX shRNA suppressed the nonoriented centrosomal movement observed with DCX shRNA alone (Fig. $3 E, F, G$; supplemental movies 3,4, available at www.jneurosci.org as supplemental material). Centrosomal motility in the coreduction condition was slightly increased compared with MARK2 shRNA alone. This result is in agreement with the partial rescue phenotype (Fig. 2).

Individual cells from each treatment were subjected to a more detailed analysis to characterize the centrosome movements (Fig. $4 A-E)$. Centrosomes in the control neurons moved in the most processive manner, and the net distances covered were the largest (Fig. 4, compare $A, B-D$ ). Their mean velocity of $24 \mu \mathrm{m} \mathrm{h}{ }^{-1}$ agrees with previously published measurements (Fishell and Hatten, 1991; Rio et al., 1997; Bovetti et al., 2007). A histogram of the displacements measured between frames ( 120 s intervals) shows a Gaussian distribution peaked at $1 \mu \mathrm{m} /$ frame. DCX shRNAtreated centrosomes showed a much wider distribution of displacements (Fig. $4 E$, red), with tails extending to very large positive (toward the pial surface) and negative [toward the ventricular zone (VZ)] values. The ratio between the total path traveled 

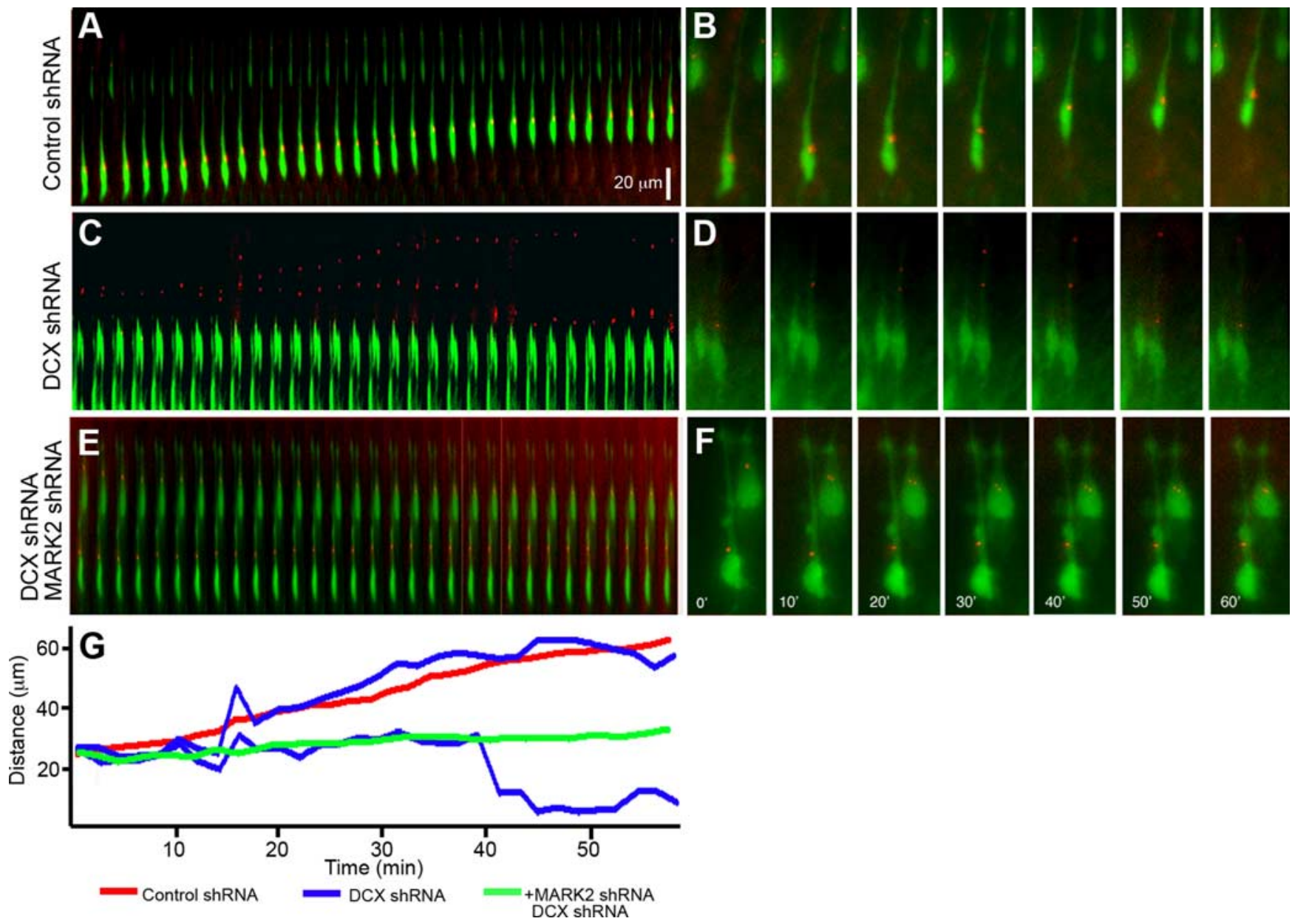

Figure 3. Analyses of centrosomal motility in organotypic brain slices from in utero electroporated brains. $\boldsymbol{A}-\boldsymbol{F}$, Time-lapse fluorescence microscopy of migrating cells in live brain slices. Cells expressing GFP with indicated shRNA; centrin-RFP (red) marks one centrosome or two separate centrioles. $\boldsymbol{A}, \boldsymbol{B}$, Control shRNA, continuous centrosomal movement preceding the nucleus; $\boldsymbol{B}$, images from $\boldsymbol{A}$ every $10^{\prime} . \boldsymbol{C}, \boldsymbol{D}, \mathrm{DCX}$ shRNA-treated cells show uncoupled movement of centrioles. $\boldsymbol{D}$, Centrosome split is visible in selected images from $\boldsymbol{C}$. $\boldsymbol{E}$, $\boldsymbol{F}$, Partial rescue of centrosomal stationary behavior is achieved by treatment with both MARK2 shRNA and DCX shRNA. $\boldsymbol{F}$, Images taken every $10^{\prime}$. $\boldsymbol{G}$, The location of the individual centrosome/centriole is each frame is plotted against time.

and the net distance that was eventually covered by each centrosome differed greatly between control and DCX shRNA-treated cells. In the control shRNA-treated cells the value was $1.1 \pm 0.1$, whereas in DCX shRNA-treated cells it was $10.6 \pm 3.9$, suggesting that the net distance traveled was smaller due to the strong tendency to reverse direction and thereby move in both orientations. The mean displacement of MARK2 shRNA-treated centrosomes was the lowest, and it differed significantly from the other groups. Cotransfection of MARK2 and DCX shRNA almost eliminated the mean displacement of the centrosomes in the negative direction. Although it differed from the MARK2 shRNA group, it did not reach control levels, again consistent with a partial rescue of the normal phenotype.

Examining the data more closely, in control cells $72 \%$ of the measured centrosome displacements were between 1 and $2 \mu \mathrm{m}$, corresponding to speeds of $0.5-1.0 \mu \mathrm{m} \mathrm{min}^{-1}$ (Fig. $4 E$, blue). The direction of movement was identical to that of the cells. In DCX shRNA-treated cells $8.9 \%$ of the centrosome displacements were oriented toward the ventricle. The fraction of events occurring at speeds exceeding $1 \mu \mathrm{m} \min ^{-1}$ reached $21 \%$, compared with only $11 \%$ in the control group. In MARK2-treated cells, $98 \%$ of displacements were measured at $1 \mu \mathrm{m}$ or less (Fig. $4 E$, yellow), indicating a net speed of $0.0-0.5 \mu \mathrm{m} \mathrm{min}{ }^{-1}$ over the frame interval. Cotransfection by MARK2 and DCX shRNA led to an increase in the fraction of displacements $>2 \mu \mathrm{m}$ from $1.8 \%$ to $5.0 \%$ (Fig. $4 E$, green). A small number of backward events were recorded in cotransfected cells, $1.5 \%$ versus none in the case of MARK2 shRNA alone. The fraction of backward events was very small compared with the case of DCX shRNA alone, consistent with the effect of MARK2 in moderating the phenotype of reduced cellular DCX. In summary, the detailed analysis demonstrates that introduction of both types of shRNA improves several specific parameters that are negatively affected by either individual shRNA.

\section{Discussion}

This study highlights a possible role of centrosomal processivity in successful radial migration in the developing cerebral cortex. Radial migration involves tight coordination between cell polarity and the cytoskeleton. This is reflected in centrosomal motility, which requires the activity of molecular motors and cytoskeletal integrity. In addition, regulation of polarity is important for coupling of the centrosome and the nucleus. The roles of MARK2/ Par-1 in cell polarity are well established (Biernat et al., 2002; Pellettieri and Seydoux, 2002; Macara, 2004). DCX is a MAP that regulates microtubule stability (Francis et al., 1999; Horesh et al., 1999). Since DCX is phosphorylated by MARK2, and this phosphorylation induces the release of DCX from microtubules, we reasoned that these two proteins could couple between the polarity and motility pathways that maintain proper neuronal migra- 


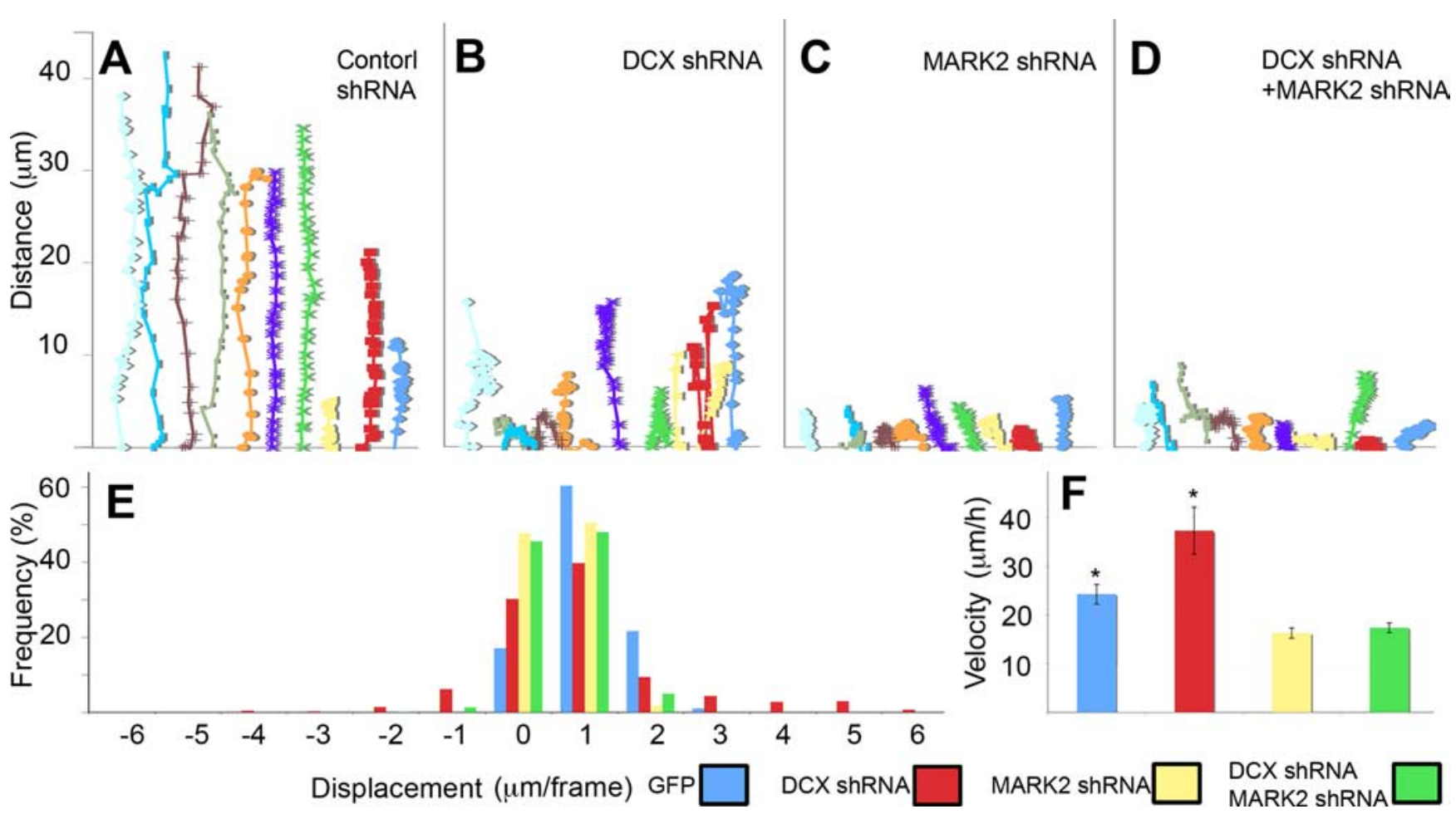

Figure 4. Analyses of centrosomal motility in organotypic brain slices from in utero electroporated brains. $A-D$, Centrosomal tracks of control and indicated shRNA treated cells in live brain slices. Centrosomes were visualized using centrin-RFP. The location of the individual centrosomes in each frame is presented: control shRNA $(\boldsymbol{A}), \mathrm{DCX}$ shRNA $(\boldsymbol{B})$, MARK2 shRNA $(\boldsymbol{C})$, DCX and MARK2 shRNA (D). $\boldsymbol{E}$, The relative displacement of each centrosome was plotted and binned according to the distance moved and the direction. The movement in the direction of the pial surface was plotted positive, while the movement toward the VZ was negative. Cells treated with DCX shRNA moved the most in both directions (red histograms), whereas MARK2 shRNA-treated cells (yellow histograms) moved the least. GFP control cells (blue histograms) moved the most in the positive orientation (1-2 $\mu \mathrm{m}$ per frame). A slight improvement is noticed in the double treated cells (green histograms). $\boldsymbol{F}$, The calculated average velocities subjected to statistical analysis. MARK2 shRNA-treated cells (yellow histogram) were significantly slower than all the other groups; a statistically significant improvement was detected in MARK2 and DCX shRNA-treated cells (green histogram). Control centrosomes (blue histogram) moved somewhat slower (not statistically different) than DCX shRNA centrosomes (red histogram). ${ }^{*} p<0.05$; error bars indicate \pm SEM.

tion. Reduction of DCX resulted in dissociated centrioles and hypermotile centrosomes, but a loss of processivity and spatial coordination with the nucleus. Reduction in immunostaining with anti-detyrosinated tubulin under the same conditions suggests that these effects may be explained by a loss of microtubule stability. In contrast, MARK2 reduction had the opposite effect, strongly impairing centrosome motility. The lack of centrosomal motility has been tied to dynein activity in the other systems (Tsai et al., 2007). Both treatments blocked neuronal migration in vivo and led to a build-up of cells at the IZ border. When the two shRNA treatments were applied simultaneously, the suppression of cell migration and that of centrosome motility were both significantly alleviated. Because DCX's phosphorylation by MARK2 releases it from microtubules (Schaar et al., 2004), an antagonistic mechanism of cellular control via MARK2 regulation of DCX can be suggested. Consequently, the removal of both elements should at least partly restore the normal phenotype. Therefore the results in this work support a coupling mechanism between cell polarity and microtubule stability via these two proteins.

Opposing effects of DCX and MARK2 have also been noted in regulation of polarity in hippocampal neurons. Reduction of DCX inhibited dendrite development (Cohen et al., 2008), whereas reduction of MARK2 stimulated their development (Terabayashi et al., 2007). A correlation between polarity and centrosome behavior has been observed in case of manipulating the levels of the key polarity protein PAR-6 which resulted in impaired migration of primary cerebellar neurons (Solecki et al., 2004). Inhibition of the cell polarity factors GSK $3 \beta$ or PKC $\zeta$ in migrating olfactory neurons resulted in impairment of centrosome reorientation and of process stabilization (Higginbotham et al., 2006). Slow motility of the centrosome and impaired coupling between the centrosome and the nucleus have been observed previously when the activity of the microtubule-associated molecular motor cytoplasmic dynein was suppressed, either directly or by interference with accessory proteins such as LIS1 and Ndel1 (Shu et al., 2004; Tsai et al., 2007). DCX is a substrate of multiple kinases, including Cdk5 (Tanaka et al., 2004b), JNK (Gdalyahu et al., 2004) and MARK2 (Schaar et al., 2004), which are all involved in regulation of neuronal migration. Of particular interest is the role of protein phosphatase 1 (PP1) in centrosomal splitting, since this phosphatase is capable of dephosphorylating DCX in a site-specific manner (Shmueli et al., 2006; Bielas et al., 2007). DCX and MARK2 are capable of mediating a cross talk between the microtubule and actin cytoskeletons (Tsukada et al., 2003, 2005; Matenia et al., 2005; Johne et al., 2008). In summary, neuronal migration coupled to seamless motility of the polarized centrosome requires a tight balance of factors involved in regulation of molecular motors and the cytoskeleton.

\section{References}

Bai J, Ramos RL, Ackman JB, Thomas AM, Lee RV, LoTurco JJ (2003) RNAi reveals doublecortin is required for radial migration in rat neocortex. Nat Neurosci 6:1277-1283.

Bielas SL, Serneo FF, Chechlacz M, Deerinck TJ, Perkins GA, Allen PB, Ellisman MH, Gleeson JG (2007) Spinophilin facilitates dephosphorylation of doublecortin by PP1 to mediate microtubule bundling at the axonal wrist. Cell 129:579-591. 
Biernat J, Wu YZ, Timm T, Zheng-Fischhöfer Q, Mandelkow E, Meijer L, Mandelkow EM (2002) Protein kinase MARK/PAR-1 is required for neurite outgrowth and establishment of neuronal polarity. Mol Biol Cell 13:4013-4028.

Bovetti S, Bovolin P, Perroteau I, Puche AC (2007) Subventricular zonederived neuroblast migration to the olfactory bulb is modulated by matrix remodelling. Eur J Neurosci 25:2021-2033.

Brann AB, Tcherpakov M, Williams IM, Futerman AH, Fainzilber M (2002) Nerve growth factor-induced p75-mediated death of cultured hippocampal neurons is age-dependent and transduced through ceramide generated by neutral sphingomyelinase. J Biol Chem 277:9812-9818.

Cohen D, Segal M, Reiner O (2008) Doublecortin supports the development of dendritic arbors in primary hippocampal neurons. Dev Neurosci 30:187-199.

des Portes V, Pinard JM, Billuart P, Vinet MC, Koulakoff A, Carrié A, Gelot A, Dupuis E, Motte J, Berwald-Netter Y, Catala M, Kahn A, Beldjord C, Chelly J (1998) A novel CNS gene required for neuronal migration and involved in X-linked subcortical laminar hetrotropia and lissencephaly syndrome. Cell 92:51-61.

Drewes G, Ebneth A, Preuss U, Mandelkow EM, Mandelkow E (1997) MARK, a novel family of protein kinases that phosphorylate microtubuleassociated proteins and trigger microtubule disruption. Cell 89:297-308.

Drewes G, Ebneth A, Mandelkow EM (1998) MAPs, MARKs and microtubule dynamics. Trends Biochem Sci 23:307-311.

Fishell G, Hatten ME (1991) Astrotactin provides a receptor system for CNS neuronal migration. Development 113:755-765.

Francis F, Koulakoff A, Boucher D, Chafey P, Schaar B, Vinet MC, Friocourt G, McDonnell N, Reiner O, Kahn A, McConnell SK, Berwald-Netter Y, Denoulet P, Chelly J (1999) Doublecortin is a developmentally regulated, microtubule-associated protein expressed in migrating and differentiating neurons. Neuron 23:247-256.

Gdalyahu A, Ghosh I, Levy T, Sapir T, Sapoznik S, Fishler Y, Azoulai D, Reiner O (2004) DCX, a new mediator of the JNK pathway. EMBO J 23:823-832.

Gleeson JG, Allen KM, Fox JW, Lamperti ED, Berkovic S, Scheffer I, Cooper EC, Dobyns WB, Minnerath SR, Ross ME, Walsh CA (1998) doublecortin, a brain-specific gene mutated in human X-linked lissencephaly and double cortex syndrome, encodes a putative signaling protein. Cell 92:63-72.

Gleeson JG, Lin PT, Flanagan LA, Walsh CA (1999) Doublecortin is a microtubule-associated protein and is expressed widely by migrating neurons. Neuron 23:257-271.

Gönczy P, Echeverri C, Oegema K, Coulson A, Jones SJ, Copley RR, Duperon J, Oegema J, Brehm M, Cassin E, Hannak E, Kirkham M, Pichler S, Flohrs K, Goessen A, Leidel S, Alleaume AM, Martin C, Ozlü N, Bork P, Hyman AA (2000) Functional genomic analysis of cell division in C. elegans using RNAi of genes on chromosome III. Nature 408:331-336.

Govek EE, Newey SE, Van Aelst L (2005) The role of the Rho GTPases in neuronal development. Genes Dev 19:1-49.

Higginbotham H, Tanaka T, Brinkman BC, Gleeson JG (2006) GSK3beta and PKCzeta function in centrosome localization and process stabilization during Slit-mediated neuronal repolarization. Mol Cell Neurosci 32:118-132.

Horesh D, Sapir T, Francis F, Wolf SG, Caspi M, Elbaum M, Chelly J, Reiner O (1999) Doublecortin, a stabilizer of microtubules. Hum Mol Genet 8:1599-1610.

Johne C, Matenia D, Li XY, Timm T, Balusamy K, Mandelkow EM (2008) Spred1 and TESK1-two new interaction partners of the kinase MARKK/ TAO1 that link the microtubule and actin cytoskeleton. Mol Biol Cell 19:1391-1403.

Kholmanskikh SS, Dobrin JS, Wynshaw-Boris A, Letourneau PC, Ross ME (2003) Disregulated RhoGTPases and actin cytoskeleton contribute to the migration defect in Lis1-deficient neurons. J Neurosci 23:8673-8681. Kholmanskikh SS, Koeller HB, Wynshaw-Boris A, Gomez T, Letourneau PC,
Ross ME (2006) Calcium-dependent interaction of Lis1 with IQGAP1 and Cdc42 promotes neuronal motility. Nat Neurosci 9:50-57.

Macara IG (2004) Parsing the polarity code. Nat Rev Mol Cell Biol 5:220-231.

Matenia D, Griesshaber B, Li XY, Thiessen A, Johne C, Jiao J, Mandelkow E, Mandelkow EM (2005) PAK5 kinase is an inhibitor of MARK/Par-1, which leads to stable microtubules and dynamic actin. Mol Biol Cell 16:4410-4422.

Moores CA, Perderiset M, Francis F, Chelly J, Houdusse A, Milligan RA (2004) Mechanism of microtubule stabilization by doublecortin. Mol Cell 14:833-839.

Pellettieri J, Seydoux G (2002) Anterior-posterior polarity in C. elegans and Drosophila-PARallels and differences. Science 298:1946-1950.

Reiner O, Carrozzo R, Shen Y, Wehnert M, Faustinella F, Dobyns WB, Caskey CT, Ledbetter DH (1993) Isolation of a Miller-Dieker lissencephaly gene containing G protein $\beta$-subunit-like repeats. Nature 364:717-721.

Rio C, Rieff HI, Qi P, Khurana TS, Corfas G (1997) Neuregulin and erbB receptors play a critical role in neuronal migration. Neuron 19:39-50.

Sapir T, Sapoznik S, Levy T, Finkelshtein D, Shmueli A, Timm T, Mandelkow EM, Reiner O (2008) Accurate balance of the polarity kinase MARK2/ Par-1 is required for proper cortical neuronal migration. J Neurosci 28:5710-5720.

Schaar BT, Kinoshita K, McConnell SK (2004) Doublecortin microtubule affinity is regulated by a balance of kinase and phosphatase activity at the leading edge of migrating neurons. Neuron 41:203-213.

Shen Y, Li N, Wu S, Zhou Y, Shan Y, Zhang Q, Ding C, Yuan Q, Zhao F, Zeng R, Zhu X (2008) Nudel binds Cdc42GAP to modulate Cdc42 activity at the leading edge of migrating cells. Dev Cell 14:342-353.

Shmueli A, Gdalyahu A, Sapoznik S, Sapir T, Tsukada M, Reiner O (2006) Site-specific dephosphorylation of doublecortin (DCX) by protein phosphatase 1 (PP1). Mol Cell Neurosci 32:15-26.

Shu T, Ayala R, Nguyen MD, Xie Z, Gleeson JG, Tsai LH (2004) Ndell operates in a common pathway with LIS1 and cytoplasmic dynein to regulate cortical neuronal positioning. Neuron 44:263-277.

Solecki DJ, Model L, Gaetz J, Kapoor TM, Hatten ME (2004) Par6alpha signaling controls glial-guided neuronal migration. Nat Neurosci 7:1195-1203.

Tanaka T, Serneo FF, Higgins C, Gambello MJ, Wynshaw-Boris A, Gleeson JG (2004a) Lis1 and doublecortin function with dynein to mediate coupling of the nucleus to the centrosome in neuronal migration. J Cell Biol 165:709-721.

Tanaka T, Serneo FF, Tseng HC, Kulkarni AB, Tsai LH, Gleeson JG (2004b) Cdk5 phosphorylation of doublecortin ser297 regulates its effect on neuronal migration. Neuron 41:215-227.

Terabayashi T, Itoh TJ, Yamaguchi H, Yoshimura Y, Funato Y, Ohno S, Miki H (2007) Polarity-regulating kinase partitioning-defective 1/microtubule affinity-regulating kinase 2 negatively regulates development of dendrites on hippocampal neurons. J Neurosci 27:13098-13107.

Timm T, Matenia D, Li XY, Griesshaber B, Mandelkow EM (2006) Signaling from MARK to tau: regulation, cytoskeletal crosstalk, and pathological phosphorylation. Neurodegener Dis 3:207-217.

Tsai JW, Chen Y, Kriegstein AR, Vallee RB (2005) LIS1 RNA interference blocks neural stem cell division, morphogenesis, and motility at multiple stages. J Cell Biol 170:935-945.

Tsai JW, Bremner KH, Vallee RB (2007) Dual subcellular roles for LIS1 and dynein in radial neuronal migration in live brain tissue. Nat Neurosci 10:970-979.

Tsukada M, Prokscha A, Oldekamp J, Eichele G (2003) Identification of neurabin II as a novel doublecortin interacting protein. Mech Dev 120:1033-1043.

Tsukada M, Prokscha A, Ungewickell E, Eichele G (2005) Doublecortin association with actin filaments is regulated by Neurabin II. J Biol Chem 280:11361-11368. 